\title{
Atrial fibrillation ablation in heart failure with reduced ejection fraction
}

\begin{abstract}
Heart failure with reduced ejection fraction (HFrEF) and atrial fibrillation (AF) are the two epidemics and present concomitantly with worse prognoses. According to the guidelines, rate control and rhythm control are the comparable selection, because rhythm control with Antiarrhythmic drugs (AADs) is not superior to rate control and anticoagulation. The CASTLE-AF trial argues the current guidelines endorse catheter ablation as first-line therapy for the treatment of AF in patients with $\mathrm{HFrEF}$ regardless of AF type. Late gadolinium enhancement magnetic resonance imaging scans (LGE-CMR) assessment has also been promising to regard predicting immediate efficacy and late outcomes after ablation. Selecting adequate AF patients with HFrEF, catheter ablation can result in significant improvement in LVEF, quality of life and functional status, with a survival benefit.
\end{abstract}

Keywords: Heart failure with reduced ejection fraction - Atrial fibrillation - Catheter ablation Antiarrhythmic drugs - LGE-CMR

\section{Abbreviations}

PABA-CHF: Pulmonary Vein Antrum Isolation vs. AV Node Ablation with BiVentricular Pacing for Treatment of Atrial Fibrillation in Patients with Congestive Heart Failure study; 6MWT: 6 Minute Walk Test; CAMTAF: Catheter Ablation vs. Medical Treatment of Atrial Fibrillation in Heart Failure; AATAC: Ablation vs. Amiodarone for Treatment of Persistent Atrial Fibrillation in Patients with Congestive Heart Failure and an Implanted Device; CAMERA-MRI: Catheter Ablation vs. Medical Rate Control in Atrial Fibrillation and Systolic Dysfunction; CASTLE-AF: Catheter Ablation vs. Standard Conventional Treatment in Patients with LV Dysfunction and AF.

\section{Introduction}

In the past 20 years, heart failure (HF) and atrial fibrillation (AF) have emerged as two new epidemics and both are expected to rise further with the aging population. AF and $\mathrm{HF}$ are associated with similar risk factors such as hypertension, diabetes, ischemic heart disease, valvular heart disease and obesity. They are often present concomitantly and their combined presentation confers a worse prognosis than either condition alone [1].

The concept of AF begets $\mathrm{HF}$ and $\mathrm{HF}$ begets AF has been well appreciated [2]. AF is a potent risk factor for adverse clinical outcomes in patients with HF with reduced ejection fraction (HFrEF). Patients with AF and HFrEF have higher mortality and hospitalization rates irrespective of which disease occurs first [3].

AF promotes HF through rapid ventricular rates, heart rate, and pulse volume irregularity, and loss of left atrial kick [4]. AF can result in LV dysfunction due to loss of atrioventricular synchrony, and as a direct cause of tachycardiainduced cardiomyopathy [5].

But in fact, HF promotes AF mainly through raised atrial filling pressures, abnormal calcium handling, neurohormonal activation, and adrenergic stimulation.

Given the poor prognosis associated with $\mathrm{AF}$ and HF, it is of importance to perform strict strategies to prevent or reverse the occurrence of AF in patients with HF and HFrEF. Antiarrhythmic drugs frequently ineffective, or not well-tolerated in patients with HFrEF, catheter ablation of $\mathrm{AF}$ provides an increasing
Yan-Rong Liu, Dian-Fu Li*

Department of Cardiology, The First Affiliated Hospital of Nanjing Medical University, 300 Guangzhou Road, Nanjing 210029, China

*Author for correspondence:

Dian-Fu Li

Email: diasy111@163.com

Received date: October 30, 2019

Accepted date: November 13, 2019

Published date: November 20, 2019 
hopeful option for rhythm control in these patients. In this review, we will summarize the rationale and current evidence for catheter ablation in patients with $\mathrm{AF}$ and HFrEF, and discusses emerging technologies, selection of patients for treatment, and future directions.

\section{Experimental}

\section{Molecular pathogenesis of AF in HFrEF}

Structural, electrophysiological and mechanical remodeling of atrial and ventricular myocardium has been associated with AF. The electrophysiological changes associated with AF include ectopic focal firing, re-entrant pathways shortening action potentials caused by atrial fibrosis which is the hallmark of structural remodeling and the main mechanism perpetuating $\mathrm{AF}$ [6].

New insights into the mechanisms underlying AF in HFrEF pathogenesis have the potential to identify new targets that could expand the available therapeutic options. Abnormal $\mathrm{Ca}^{2+}$ dynamics, neurohormonal dysregulation, inflammation and microRNA markers of structural or electrical remodeling are deduced to the most important mechanisms [7].

\section{AF induced cardiomyopathy or cardiomyopathy superimposed AF?}

Arrhythmia induced cardiomyopathy (AIC) is a common kind of cardiomyopathy. There are two types of AIC. Type 1 AIC: Arrhythmia induced. This is when arrhythmia is solely responsible for AIC and LV function returns to normal upon successful treatment of arrhythmia. Type 2 AIC: Arrhythmia mediated. Arrhythmia exacerbates the underlying cardiomyopathy and treatment of the arrhythmia results only in partial resolution of the cardiomyopathy [8].

$\mathrm{AF}$ is the most common reason for AIC. AF is known to trigger a reversible dilated cardiomyopathy referred to as AF-induced cardiomyopathy. In these patients, restoration of sinus rhythm such as catheter ablation can cure HF obviously. The reversal of cardiomyopathy by the elimination of AF confirms AF-induced cardiomyopathy, and also, its prognosis is improved.

But in other circumstances, AF is not the only reasoninduced HF (such as ischemic, infiltrative, or toxic/ drug-related) should benefit from the restoration of sinus rhythm? In clinical medicine, a frequent challenge is to identify a superimposed AF worsens known cardiomyopathy. So, it is of paramount importance to determine whether or to what extent $\mathrm{AF}$ contributes to HFrEF.

\section{Treatment of AF in HFrEF}

Antiarrhythmic drugs (AADs) therapy vs. catheter ablation

The main recommended goals of therapy for patients with $\mathrm{AF}$ in the HFrEF patients continue to focus on the prevention of thromboembolism and symptom relief. The initial treatment of AF in HFrEF should include optimization of guideline-directed medical therapy (beta-blockers, angiotensin-converting enzyme inhibitors or angiotensin receptor blockers, diuretic agents and aldosterone blockers) to optimize reverse remodeling. The aim of antiarrhythmic drug therapy is to improve AF-related symptoms. The efficacy of AADs to maintain sinus rhythm is modest, on the otherwise, AADs induced pro-arrhythmia or extracardiac side effects are frequent [9]. Dofetilide and amiodarone are the only guideline-recommended antiarrhythmic drugs for the treatment of AF patients with HFrEF. The current American and European guidelines for the management of patients with $\mathrm{AF}$ and $\mathrm{HFrEF}$ relied heavily on the DIAMOND-CHF and AF-CHF trials, which found no difference in mortality between pharmacological rhythm control and rate control. Therefore, according to the guidelines, rate control and rhythm control are the comparable selection for both. There are several possible explanations for this discrepancy. First, AADs may not be efficacious in maintaining sinus rhythm in patients with HFrEF. Another reason for such a lack of clinical benefit of pharmacologic rhythm control may be in part due to the significant toxicity that is associated with antiarrhythmic drugs. Lastly, Dofetilide and amiodarone both pose risks and have multiple drug-drug interactions to affect the whole benefit [10]. Hence, the decision to initiate long-term AADs therapy needs to balance symptom burden, possible adverse drug reactions, and patient preferences.

\section{Catheter Ablation}

The ablation of $\mathrm{AF}$ has evolved from a rare and complex procedure to a common electrophysiological technique. AF catheter ablation is highly efficacious for relieving symptoms and treating drug-refractory AF. However, the majority of randomized controlled trials (RCTs) of drug therapy vs. catheter ablation have enrolled patients with preserved ventricular function [11]. Previous studies have reported that maintenance of sinus rhythm by catheter ablation can significantly improve LV function. Some small-scale RCTs of AF patients with HFrEF suggested that successful rhythm control by catheter ablation improved cardiac function and was associated with significant reduction rates of 
stroke and mortality [12]. But these RCTs to assess the efficacy of catheter ablation were small, and none had assessed mortality as a primary endpoint. "Hard endpoints" require large numbers of patients with extensive follow-up [13].

Fortunately, to date, two larger RCTs assessing the efficacy of AF ablation in patients with HFrEF have been completed, one is AATAC-AF (Ablation vs. Amiodaron for Treatment of Atrial Fibrillation in Patients with Congestive Heart Failure and Implanted ICD/CRT-D), which randomized 203 patients with $\mathrm{AF}$ and $\mathrm{HFrEF}$ to either amiodarone or catheter ablation, the primary endpoint was freedom of AF during follow-up. The trial proved that ablation is superior to amiodarone in terms of rhythm control and an improvement in LV dysfunction [14].

Another RCT published in 2018 was CASTLE-AF trial (Catheter ablation for atrial fibrillation with Heart Failure), which enrolled 363 patients with paroxysmal or persistent AF and HFrEF. Subjects were randomly assigned to either catheter ablation or pharmacological rate control with median follow up of 37.8 months. On the other hand, the authors evaluated the hard endpoints of death and HF hospitalizations; the results showed catheter ablation was superior to conventional medical treatment of either rate or rhythm control [15].

However, the large size in a modest trial, large loss to follow-up in the ablation arm, highly selective population recruited, and lack of a blinded intervention along with lack of data on uptitration of medical therapy dosing in the two arms over time is a potential source of bias that may have favored the intervention arm and remains unresolved. Patients who were randomly assigned to medical therapy were encouraged to precede with pharmacologic rhythm control with the use of class IA, class IC, and class III agents, many of which can increase mortality among patients with systolic HF [16].

CABANA (Catheter Ablation vs. Anti-arrhythmic Drug Therapy for Atrial Fibrillation Trial) trial is another RCTs that has been published. A total of 2204 symptomatic patients with AF were enrolled, and the median follow-up was 48.5 months. In the intention to -treat analysis, catheter ablation was associated with lower but no significant rate of composite mortality, disabling stroke, serious bleeding, or cardiac arrest.

But in CABANA trial, there are only $15 \%$ of the AF patients enrolled in the had HF (also HFrEF and $\mathrm{HFpEF}$ ) at the time of randomization, and primary endpoint events were observed in only 49 patients. Given the smaller number of the patients, the effects of catheter ablation are the subgroup with HFrEF have not been analyzed, so the trial is not likely to add meaningfully evidence [17].

Notably, a recent AF guideline update only provides class IIB indications for catheter ablation for AF-HF. Given this information, the CASTLE-AF trial argues the current guidelines endorse catheter ablation as firstline therapy for the treatment of AF in patients with HFrEF regardless of AF type. The debate on whether $\mathrm{AADs}$ or catheter ablation for rhythm control is the better strategy for managing AF complicating HFrEF continues. Further trials are required (Table 1).

\begin{tabular}{|c|c|c|c|c|c|c|c|c|}
\hline No & Year & Study & $\begin{array}{l}\text { Patients' } \\
\text { Number }\end{array}$ & Comparison arm & $\begin{array}{l}\text { Primary } \\
\text { outcomes }\end{array}$ & $\begin{array}{l}\text { Secondary } \\
\text { outcomes }\end{array}$ & $\begin{array}{l}\text { Follow-up } \\
\text { (months) }\end{array}$ & Results \\
\hline 1 & 2008 & $\begin{array}{l}\text { PABA- } \\
\text { CHF[18] }\end{array}$ & 81 & $\begin{array}{l}\text { PVI vs } \\
\text { atrioventricular- } \\
\text { node ablation with } \\
\text { biventricular pacing }\end{array}$ & $\begin{array}{l}\text { Minnesota } \\
\text { Living score and } \\
6 \mathrm{MWT}\end{array}$ & - & 6 & $\begin{array}{l}\text { PVI was superior to } \\
\text { Atrioventricualr-node ablation } \\
\text { with biventricular pacing }\end{array}$ \\
\hline 2 & 2013 & $\begin{array}{l}\text { CAMATF } \\
{[19]}\end{array}$ & 52 & $\begin{array}{l}\text { Catheter ablation vs } \\
\text { rate control }\end{array}$ & LVEF & \begin{tabular}{|l|} 
Peak oxygen \\
consumption \\
and Minnesota \\
living score \\
\end{tabular} & 6 & $\begin{array}{l}\text { Catheter ablation was effective } \\
\text { in restoring sinus rhythm and } \\
\text { can improve LV function and } \\
\text { HF symptoms }\end{array}$ \\
\hline 3 & 2016 & $\begin{array}{l}\text { AATAC } \\
{[14]}\end{array}$ & 203 & $\begin{array}{l}\text { Catheter ablation vs } \\
\text { amiodarone }\end{array}$ & $\begin{array}{l}\text { Recurrence } \\
\text { of AF }\end{array}$ & \begin{tabular}{|l|} 
All-cause \\
mortality and \\
hospitalization \\
\end{tabular} & 24 & $\begin{array}{l}\text { Catheter was superior to } \\
\text { amiodarone in primary and } \\
\text { secondary outcomes }\end{array}$ \\
\hline 4 & 2017 & $\begin{array}{l}\text { CAMERA- } \\
\text { MRI [20] }\end{array}$ & 52 & $\begin{array}{l}\text { Catheter ablation vs } \\
\text { rate control }\end{array}$ & $\begin{array}{l}\text { Peak oxygen } \\
\text { consumption }\end{array}$ & $\begin{array}{l}\text { Quality of life, } \\
\text { BNP,6MWT } \\
\text { and EF }\end{array}$ & 12 & $\begin{array}{l}\text { Catheter ablation was } \\
\text { superior to rate control in } \\
\text { exercise performance and HF } \\
\text { symptoms and neurohormone } \\
\text { status }\end{array}$ \\
\hline 5 & 2018 & $\begin{array}{l}\text { CASTLE- } \\
\text { AF [15] }\end{array}$ & 363 & $\begin{array}{l}\text { Catheter ablation vs } \\
\text { AADs }\end{array}$ & $\begin{array}{l}\text { death and HF } \\
\text { hospitalization }\end{array}$ & - & 37.8 & $\begin{array}{l}\text { Catheter ablation was superior } \\
\text { to AADs in primary outcomes }\end{array}$ \\
\hline
\end{tabular}




\section{The procedure of catheter ablation}

Pulmonary vein isolation (PVI) vs. AV-node ablation plus bi-ventricular pacing: Choices of catheter ablation strategy (PVI vs. AV-node ablation) trials in HF have questioned. The standard-of-care ablation treatment for AF is PVI, which encircles the pulmonary veins with lesions, preventing abnormal electric signals generated in the veins from invading the atria. PABA-CHF trial compared PVI to AV-node ablation plus bi-ventricular pacing for the treatment of HF patients with HFrEF, 6-minute walk distance and Minnesota Living with HF questionnaire. The study showed PVI to be superior to AV node ablation and bi-ventricular pacing with respect to the primary endpoint [18-21]. So, atrioventricular node ablation and subsequent biventricular pacing may be perceived as an extreme form of rate control in AF management. Guidelines recommend atrioventricular nodal ablation with pacing only when rate or rhythm control is not achievable by pharmacological therapy (Class IIa, Level of Evidence: B). Catheter ablation is currently the cornerstone of rhythm control approaches in AF patients with HFrEF [22]. Moreover, in the past 5 years, the technology and modality of catheter ablation have dramatically changed. New advanced irrigated catheter tips and new modules, such as contact force monitoring systems, may have resulted in the relatively high success rate of catheter ablation for AF in HFrEF, which likely contributed to improvements in patientcentered outcomes [23].

The possible harmfulness of catheter ablation: However, in patients with the persistent form of $\mathrm{AF}$, who typically develop fibrotic remodeling in the atria, the mechanisms giving rise to AF shift from electrical abnormality in the pulmonary veins to re-circulating electrical waves perpetuated by the fibrotic substrate. All attempts to target the arrhythmogenic sources in the fibrotic substrate, such as execution of linear ablation lesions across the left atrial loof and mitral valve isthmus, and ablation of complex fractionated atrial electrograms, have failed to deliver reasonable outcomes [24]. Therefore, although ablation seeks to restore atrial contractile function, the ability of the LA to transport pulmonary venous blood is decreased by the procedure, especially long-standing AF and preexisting LA fibrosis. Efforts to interrupt neural circuits with catheter ablation can cause further injury to the LA, as evidenced acutely, by cardiomyocyte necrosis and tissue edema, and chronically, by the loss of contractile and reservoir function as a result of replacement fibrosis. The extent of these changes depends on the number of ablation lines and ablation procedures.
The quality of the procedure is paramount and should be a critical appraisal. And given the invasive nature of the procedure, catheter ablation carries certain procedure risks, including stroke, pericardial effusion, atrioesophageal fistula, bleeding, pulmonary stenosis, pneumonia, and rarely death.

The selection of catheter ablation patients: Balancing the benefits and harmfulness of catheter ablation, selecting which AF patients with HFrEF is of very importance to affect the patients' prognosis and safeness. Before the catheter procedure, we should evaluate the patients' status seriously. Procedures should be performed by well-trained electrophysiologists in experienced centers. Therapeutic choice depends on the culprit arrhythmia, patient comorbidities and preferences. And how do we characterize patients who are optima candidates for ablation? Clinical markers based on aggregated risk scores need to be investigated, including other marker, such as genetic markers and biochemical markers. Recently, a large sample size study from Germany which enrolled more than $1400 \mathrm{AF}$ patients undergoing first $\mathrm{AF}$ ablation demonstrated that $\mathrm{AF}$ recurrence post ablation can be predicted using the APPLE score including 'Age $>65$ years', 'persistent AF', 'imPaired eGFR $\left(<60 \mathrm{~mL} / \mathrm{min} / 1.73 \mathrm{~m}^{2}\right)$, 'Left atrial diameter $\geq 43 \mathrm{~mm}$ ', and 'Ejection fraction $<50 \%$, have increased AF recurrence risk [25].

Structural tissue remodeling, including increased collagen disposition, loss of myocytes and fibrosis, have been shown to be related to AF with HFrEF patients. Late gadolinium enhancement magnetic resonance imaging scans (LGE-CMR) allows to characterize left atrial anatomy, structural remodeling; and to detect and quantify myocardial fibrosis, and LGE-CMR has been extensively validated in histopathologic studies and potentially helping to better select patients for this therapy [26]. LGE-CMR assessment has also been promising to regard predicting immediate efficacy and late outcomes after ablation.

\section{Results and Discussion}

In general, extensive atrial remodeling has been shown to predict worse outcomes after ablation procedures: patients with high LA scar burden, increased LA sphericity, and LA volume, as well as incomplete ablation lines, have increased AF recurrence risk [27]. The benefit of AF ablation was most apparent in patients without ventricular fibrosis as assessed by LGE-CMR. LGE-CMR has been used to estimate chronic lesion scarring after radiofrequency ablation in 
the LA [28]. Pre-ablation LGM-CMR might identify patients with heavy burdens of scar who are unlikely to respond to ablation. But these techniques must become reproducible and reliable and must be assessed in multicentre trials.

According to these clinical circumstance, the ideal patient with the highest likelihood of procedural success is one with recent-onset $\mathrm{HF}$, recent-onset $\mathrm{AF}$ with fast ventricular rates, idiopathic CMP, ventricular LGEMRI negative, lower LVEF, LA enlargement (less than $55 \mathrm{~mm}$ ), LA fibrosis less than $10 \%$, and young patients (less than 65 years), and no or few comorbidities[29]. Meanwhile, the aggressive management of cardiovascular risk factors (hypertension, diabetes mellitus, sleep disorders, obesity, excessive alcohol, smoking) and cardiac rehabilitation after ablation reduces the odds of recurrent $\mathrm{AF}$ and is an important element in the quality of care.

\section{Conclusion}

In conclusion, the present evidence of clinical trials suggests that PVI catheter ablation compared to AADs for AF in patients with $\mathrm{HFrEF}$ results in significant improvement in LVEF, quality of life and functional status, with a survival benefit. But it also remains imperative to design large "hard endpoints" RCTs that reflect shifting global ablation techniques, technologies, and patient selection.

But questions remain. Do the benefits of catheter ablation in HFrEF also extend to patients with AF and HFpEF? Who benefits most from an ablation strategy? Finally, in those who may benefit from catheter ablation, when is the optimal time to intervene and when is it too late? Figure 1 shows our opinions in managing rhythm in AF patients with HFrEF. Currently, some ongoing RCTs; such as the RAFT-AF, CATCH-AF, AMICA and AFARC-LVF trials will be completed in 2019, which will further improve our understanding of the utility of catheter ablation in AF patients with HFrEF.

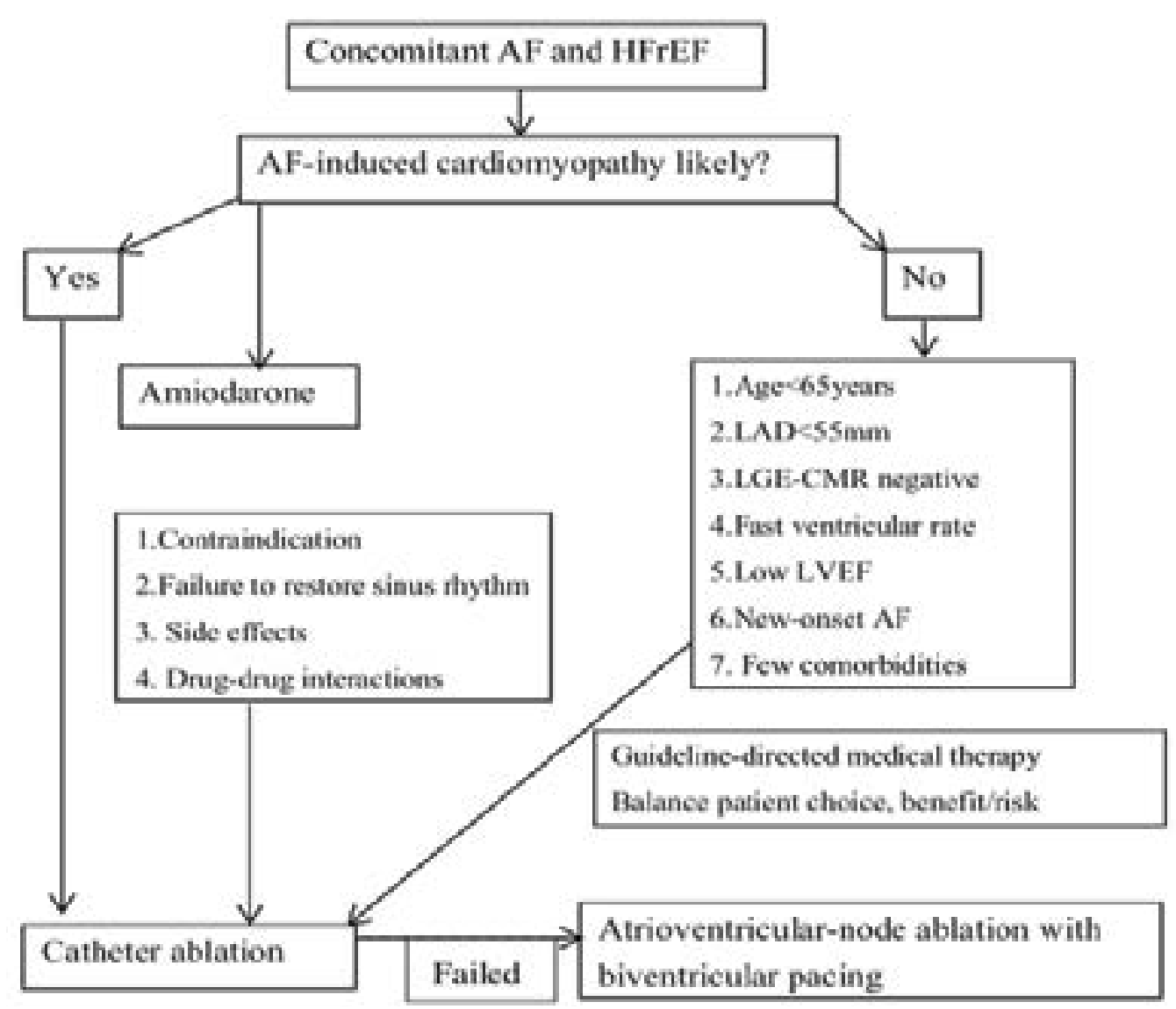

Figure 1: Proposed rhythm managing in patients with AF in HFrEF. 


\section{References}

1. Santhanakrishnan R, Wang N, Larson MG, et al. Atrial fibrillation begets heart failure and vice versa: temporal associations and differences in preserved versus reduced ejection fraction. Circulation. 2016;133(5):484-492.

2. Richter S, Di Biase L, Hindricks G. Atrial fibrillation ablation in heart failure. Eur Heart J. 2019;40(8): 663-671.

3. Kirchhof P, Benussi S, Kotecha D, et al. 2016 ESC Guidelines for the management of atrial fibrillation developed in collaboration with EACTS. Eur Heart J. 2016;37(38):2893-2962.

4. Chen S, Purerfellner H, Meyer C, et al. Rhythm control for patients with atrial fibrillation complicated with heart failure in the contemporary era of catheter ablation: a stratified pooled analysis of randomized data. Eur Heart J. 2019.

5. Agasthi P, Tseng A, Lee JZ, et al. Atrial fibrillation ablation should be first-line therapy in patients with heart failure reduced ejection fraction. Cardiol Clin. 2019;37(2):185-195.

6. Rosenberg JH, Werner JH, Plitt GD, et al. Immunopathogenesis and biomarkers of recurrent atrial fibrillation following ablation therapy in patients with preexisting atrial fibrillation. Expert Rev Cardiovasc Ther. 2019;17(3):193-207.

7. Luo X, Yang B, Nattel S. MicroRNAs and atrial fibrillation: Mechanisms and translational potential. Nat Rev Cardiol. 2015;12(2):80-90.

8. Huizar JF, Ellenbogen KA, Tan AY, et al. Arrhythmia-induced cardiomyopathy: JACC State-of-the-art review. J Am Coll Cardiol. 2019;73(18):2328-2344.

9. Baher A, Marrouche NF. Treatment of atrial fibrillation in patients with co-existing heart failure and reduced ejection fraction: Time to revisit the management guidelines? Arrhythm Electrophysiol Rev. 2018;7(2):91-94.

10. Carlisle MA, Fudim M, DeVore AD, et al. Heart failure and atrial fibrillation, like fire and fury. JACC Heart Fail. 2019;7(6):447456.

11. Reddy YNV, Borlaug BA, O'Connor CM, et al. Novel approaches to the management of chronic systolic heart failure: future directions and unanswered questions. Eur Heart J. 2019.

12. Virk SA, Bennett RG, Chow C, et al. Catheter ablation versus medical therapy for atrial fibrillation in patients with heart failure: a meta-analysis of randomised controlled trials. Heart Lung Circ. 2019;28(5):707-718.

13. Catheter Ablation for atrial fibrillation with heart failure. New Eng J Med. 2018;379(5):490-492.

14. Di Biase L, Mohanty P, Mohanty S, et al. Ablation versus amiodarone for treatment of persistent atrial fibrillation in patients with congestive heart failure and an implanted device: Results from the AATAC multicenter randomized trial. Circulation. 2016;133(17):1637-1644.

15. Marrouche NF, Brachmann J, Andresen D, et al. Catheter ablation for atrial fibrillation with heart failure. N Engl J Med. 2018;378(5): 417-427.
16. Packer M. Electrophysiological interventions in the treatment of chronic heart failure: a comparison of the strength of evidence supporting cardiac resynchronization for electrical conduction delay and catheter ablation for atrial fibrillation. Eur J Heart Fail. 2019;21(4): 398-401.

17. Mark DB, Anstrom KJ, Sheng S, et al. Effect of catheter ablation vs medical therapy on quality of life among patients with atrial fibrillation: The CABANA randomized clinical trial. JAMA. 2019;321(13):1275-1285.

18. Khan MN, Jaïs P, Cummings J, et al. Pulmonary-vein isolation for atrial fibrillation in patients with heart failure. N Engl J Med. 2008;359:1778-1785.

19. Jones DG, Haldar SK, Hussain W, et al. A randomized trial to assess catheter ablation versus rate control in the management of persistent atrial fibrillation in heart failure. J Am Coll Cardiol. 2013;61(18):1894-1903.

20. Prabhu S, Taylor AJ, Costello BT, et al. Catheter ablation versus medical rate control in atrial fibrillation and systolic dysfunction: The CAMERA-MRI study. J Am Coll Cardiol. 2017;70(16):1949-1961.

21. Khan MN, Jaï P, Cummings J, et al. Pulmonary-vein isolation for atrial fibrillation in patients with heart failure. $\mathrm{N}$ Engl J Med. 2008;359:1778-85.

22. Vrachatis D, Deftereos S, Kekeris V, et al.. Catheter ablation for atrial fibrillation in systolic heart failure patients: stone by stone, a castle. Arrhythm Electrophysiol Rev. 2018;7(4):265-272.

23. Boyle PM, Zghaib T, Zahid S, et al. Computationally guided personalized targeted ablation of persistent atrial fibrillation. Nat Biomed Eng. 2019.

24. Packer M. Effect of catheter ablation on pre-existing abnormalities of left atrial systolic, diastolic, and neurohormonal functions in patients with chronic heart failure and atrial fibrillation. Eur Heart J. 2019;40(23):1873-1879.

25. Kholmovski EG, Morris AK, Chelu MG. Cardiac MRI and fibrosis quantification. Card Electrophysiol Clin. 2019;11(3):537-549.

26. Almeida AG. Searching for the key to foreseeing left atrial fibrosis in atrial fibrillation: could this be simple and reliable with MRI? Radiology. 2019;292(3):583-584.

27. Margulescu AD, Nunez-Garcia M, Alarcon F, et al. Reproducibility and accuracy of late gadolinium enhancement cardiac magnetic resonance measurements for the detection of left atrial fibrosis in patients undergoing atrial fibrillation ablation procedures. Europace. 2019;21(5):724-731.

28. Chelu MG, King JB, Kholmovski EG, et al. Atrial fibrosis by late gadolinium enhancement magnetic resonance imaging and catheter ablation of atrial fibrillation: 5-year follow-up data. J Am Heart Assoc. 2018;7(23):e006313.

29. Link MS, Haissaguerre M, Natale A. Ablation of atrial fibrillation: patient selection, periprocedural anticoagulation, techniques, and preventive measures after ablation. Circulation. 2016;134(4):339-352. 